\title{
CP Violation reach of EC beams
}

\section{Catalina Espinoza* and Jose Bernabeu}

IFIC, Universitat de València-CSIC, E-46100, Burjassot, València, Spain

E-mail: m. catalina.espinoza@uv.es, jose.bernabeu@uv.es

The ultimate goal for future neutrino facilities is the determination of $\mathrm{CP}$ violation in neutrino oscillations. Besides $|U(e 3)| \neq 0$, this will require precision experiments with a very intense neutrino source and energy control. With this objective in mind, the creation of monochromatic neutrino beams from the electron capture decay of boosted ions by the SPS of CERN has been proposed. We discuss the capabilities of such a facility as a function of the energy of the boost and the baseline for the detector. We compare the physics potential for two different configurations: I) $\gamma=90$ and $\gamma=195$ (maximum achievable at present SPS) to Frejus; II) $\gamma=195$ and $\gamma=440$ (maximum achievable at upgraded SPS) to Canfranc. We conclude that the SPS upgrade to 1000 $\mathrm{GeV}$ is important to reach a better sensitivity to $\mathrm{CP}$ violation iff it is accompanied by a longer baseline. In both Setups, the gain in the $\mathrm{CP}$ violation sensitivity with a previous knowledge of $|U(e 3)|$ is apparent.

10th International Workshop on Neutrino Factories, Super beams and Beta beams June 30 - July 52008

Valencia, Spain

\footnotetext{
${ }^{*}$ Speaker.
} 


\section{Introduction}

Neutrino oscillation phenomena are energy dependent for a fixed distance between source and detector, and the observation of this energy dependence would disentangle the two important parameters: whereas $|U(e 3)|$ gives the strength of the appearance probability, the CP phase $\delta$ acts as a phase-shift in the interference pattern. These properties suggest the consideration of a facility able to study the detailed energy dependence by means of fine tuning of a boosted monochromatic neutrino beam. In an EC facility the neutrino energy is dictated by the chosen boost of the ion source and the neutrino beam luminosity is concentrated at a single known energy which may be chosen at will for the values in which the sensitivity for the $\left(\theta_{13}, \delta\right)$ parameters is higher. The study of neutrino oscillations in terms of neutrino energy will be able to separate out the CP phase $\delta$ from the mixing parameters. A control of this energy may be obtained from the choice of the boost in the EC facility with a single ion. We discuss the proposal of EC beams of short-lived ions [1].

\section{Physics reach at different energies and baselines}

For the study of the physics reach associated with such a facility, we combine two different energies for the same ${ }^{150} D y$ ion in each of two Setups [2]. In all cases we consider $10^{18}$ decaying ions/year, a water Cerenkov Detector with fiducial mass of 440 Kton and both appearance $\left(v_{\mu}\right)$ and disappearance $\left(v_{e}\right)$ events. Setup I is associated with a five year run at $\gamma=90$ (close to the minimum energy to avoid atmospheric neutrino background) plus a five year run at $\gamma=195$ (the maximum energy achievable at present SPS), with a baseline $L=130 \mathrm{Km}$ from CERN to Frejus. The results for Setup I are going to be compared with those for Setup II, associated with a five year run at $\gamma=195$ plus a five year run at $\gamma=440$ (the maximum achievable at the upgraded SPS with proton energy of $1000 \mathrm{GeV}$ ), with a baseline $L=650 \mathrm{Km}$ from CERN to Canfranc.

As observed in Fig. 1, the principle of an energy dependent measurement (illustrated here with two energies) is working to separate out the two parameters. For the Setup I the precision obtainable for the mixing is much better than that for the CP phase. As seen, even mixings of $1^{\circ}$ are still distinguishable.

In the case of Setup II the longer baseline for $\gamma=195$ leads to a value of $E / L$ well inside the second oscillation. In that case the associated strip in the $\left(\theta_{13}, \delta\right)$ plane has a more pronounced curvature, so that the two parameters can be better disentangled. Qualitatively, one notices that the precision reachable for the $\mathrm{CP}$ phase is better than that in the case of Setup I.

The corresponding $(3 \sigma)$-sensitivities for a $\mathrm{CP}$ violation discovery, i. e., $\delta \neq 0,180^{\circ}$, are compared in Fig.2 (left) for both Setups. In both cases, $\theta_{13}$ and $\delta$ are previously unknown, so that we make the analysis for a two-parameter fit. It is worth to notice the superiority of Setup II, in observing CP violation at smaller values of $\theta_{13}$ as well as in the capability of distinguishing a value of the CP phase $\delta$ closer to $0^{\circ}$ or $180^{\circ}$, for a given $\theta_{13}$.

If $\theta_{13}$ is previously known, as expected if $\theta_{13} \gtrsim 3-4^{\circ}$, the corresponding analysis for the sensitivity to discover CP violation is presented in Fig. 2 (right). In this case, the $\chi^{2}$ fit is made with the single parameter $\delta$. One may notice that the improvement in this sensitivity for the same Confidence Level is impressive, as compared with Fig. 2 (left). If $\theta_{13}$ is large enough, going step by step in the determination of the neutrino oscillation parameters by means of several generation 

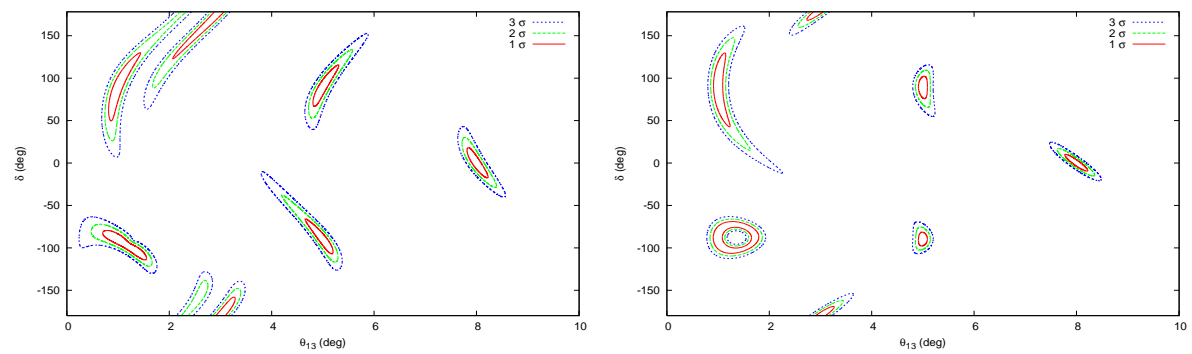

Figure 1: Two-parameter fit for $\left(\theta_{13}, \delta\right)$ from statistical distribution of events with assumed values of the parameters. Setup I (left) and Setup II (right) as explained in the text.
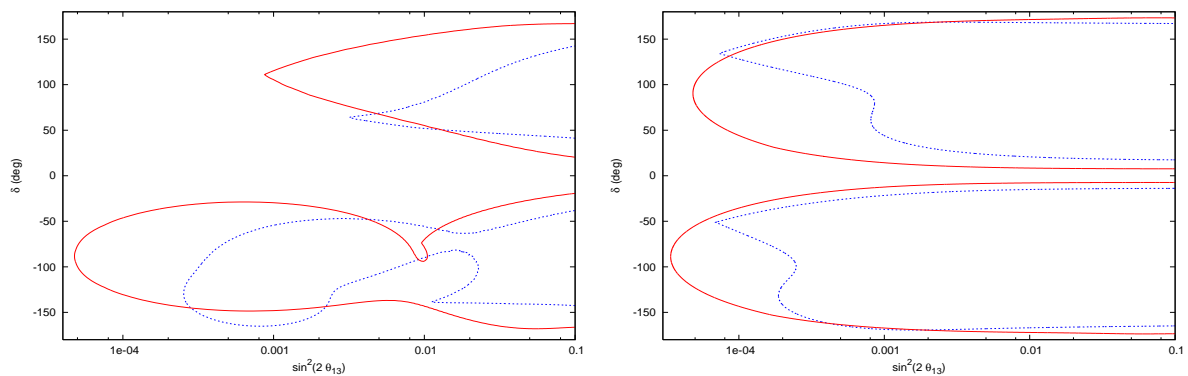

Figure 2: $\mathrm{CP}$ violation exclusion plot at $99 \% \mathrm{CL}$, if $\theta_{13}$ is still unknown (left) and if $\theta_{13}$ were previously known (right), for the two reference Setups: I (broken blue line) and II (continuous red line).

experiments is very rewarding. As in Fig. 2 (left), Setup II provides better sensitivity to the discovery of CP violation than Setup I. For Setup II, the result would be so good that it would enter into the regime of a precision experiment.

\section{Conclusions}

The simulations of the physics output for an EC neutrino beam at different energies indicate that the principle of energy dependence for separating out the CP-even and CP-odd contributions to the neutrino oscillation probability works. The upgrade to higher energy in the SPS boost $\left(E_{p}=\right.$ $1000 \mathrm{GeV}$ ) helps to have a better sensitivity to $\mathrm{CP}$ violation, which is the main objective of the next generation neutrino oscillation experiments, iff accompanied by a longer baseline. The best $E / L$ in order to have a higher sensitivity to the mixing $|U(e 3)|$ is not the same as that for the CP phase $\delta$. Like the phase-shifts in interference phenomena, the presence of $\delta$ is easier to observe when the energy of the neutrino beam enters into the region of the second oscillation. Instead, the mixing is better seen around the first oscillation maximum. If $\theta_{13} \gtrsim 3-4^{\circ}$, the previous knowledge on the connecting mixing $\theta_{13}$ would greatly improve the sensitivity to $\mathrm{CP}$ violation discovery in this facility.

\section{References}

[1] J. Bernabeu, J. Burguet-Castell, C. Espinoza and M. Lindroos, JHEP 0512, 014 (2005).

[2] J. Bernabeu and C. Espinoza, Phys. Lett. B 664, 285 (2008). 